\title{
Déformation de la houle sur une plage à barres : Sète (Campagne PNEC automne 2000)
}

\author{
R . Certain*, C. Dulou**. \\ * Doctorant, Laboratoire de Sédimentologie Marine, Université de Perpignan, 52 av. de \\ Villeneuve, 66000 Perpignan. Téléphone: 0468661745 \\ Courrier électronique : certain@univ-perp.fr \\ ** ATER, Département de Géologie et Océanographie, UMR 5805-EPOC, Université de \\ Bordeaux I, 33405 Talence, cedex. Téléphone:05 568488 35. Courrier électronique: \\ c.dulou@epoc.u-bordeaux.fr
}

Résumé

Une analyse de l'évolution cross-shore de la houle à l'approche de la côte est effectuée sur un site comportant deux barres sédimentaires parallèles à la plage. L'analyse spectrale de données acquises sous plusieurs situations d'agitations est réalisée. Les transferts d'énergie dus à des interactions non-linéaires onde-onde qui s'opèrent au cours de situations caractéristiques sont décrits.

Abstract

A cross-shore study of the evolution of waves is made near a barred-beach. A spectral analysis of several hydrodynamic condition measurements is performed by Fast Fourier Transform algorithm. Non linear energy transfers between the different bands of the spectral domain are described.

\section{1-Introduction}

L'hydrodynamique complexe induite par la houle autour de la zone de déferlement a connu des développements récents importants tant dans sa modélisation numérique (par exemple Abadie, 1998 ; Madsen et al., 1997; Cadène, 2000) que dans l'analyse de ses processus physiques en environnements simplifiés comme les canaux à houle (Dulou, 2000a, O'Hare et Davis, 1993). Mais cela ne doit pas masquer les grandes lacunes quant à l'analyse en milieu naturel (Holman and Sallenger, 1993). Le but de ce travail est de contribuer à cette connaissance de l'hydrodynamique côtière (Elgar et al., 1997) dans la zone de déferlement sur une plage à barres.

Des études simplifiées en canal à houle (houle monochromatique ou bichromatique) de formation de barres sédimentaires littorales (Dulou et al., 2000b) montrent que la formation des barres semble liée à l'action du premier harmonique (le plus énergétique) et aux interactions non-linéaires onde-onde. Une houle est composée d'harmoniques libres, par exemple de fréquences $\mathrm{f} 1$ et $\mathrm{f} 2$, et d'harmoniques liés, qui sont égaux à $\mathrm{n}$ fois la fréquence du pic principal à laquelle elles sont liées, soient $f 1 a=2 f 1, f 1 b=3 f 1$ et $f 2 a=2 f 2, f 2 b=3 f 2$ etc. Les interactions entre les harmoniques libres, qui se traduisent par des transferts d'énergie entre les différentes composantes de l'onde, produisent des sous-harmoniques (interaction différence, $\mathrm{fl}-\mathrm{f} 2$ ) et des super-harmoniques (interaction somme, $\mathrm{f} 1+\mathrm{f} 2$ ). Ces interactions nonlinéaires onde-onde augmentent alors que la profondeur diminue. Dans la zone de shoaling (frottement sur le fond provoquant ralentissement et asymétrie de la vague) jusqu'au point de déferlement les transferts d'énergie se font plutôt vers les harmoniques supérieurs $(\mathrm{fl}+\mathrm{f} 2)$ et 


\section{VII ${ }^{\grave{m} e s}$ Journées Nationales Génie Civil - Génie Côtier, Anglet, France, 15-17 Mai 2002}

les harmoniques liées. A partir du point de déferlement les transferts se font au profit des basses fréquences (f1-f2) que l'on retrouve dans la zone de jet de rive, à la côte, car les hautes fréquences s'atténuent les premières.

Ces phénomènes très étudiés en canal, sont ici analysés en nature grâce au profil instrumenté. La houle au large sur le site retenu ayant une direction quasiment normale à la plage (l'obliquité maximale étant de $20^{\circ}$ ), le but est de suivre l'évolution cross-shore de la houle à l'approche de la côte et au passage de chacune des deux barres présentes.

Les descriptions de la zone d'étude, du dispositif expérimental et de la méthode d'analyse seront exposés dans la première partie. Une analyse de l'effet des barres sur la houle est effectuée ensuite. Leur impact lors de plusieurs situations de tempête est étudié, ainsi que les transferts d'énergie qui s'opèrent au cours de situations caractéristiques.

\section{2-Méthodologie}

\subsection{Présentation du site}

La zone retenue pour cette expérience se situe à Sète, dans le Golfe du Lion, en Languedoc-Roussillon (fig.1). Elle correspond au site méditerranéen de l'ART 7 du PNEC (Programme National d'Environnement Côtier), qui étudie les environnements à barres sédimentaires.

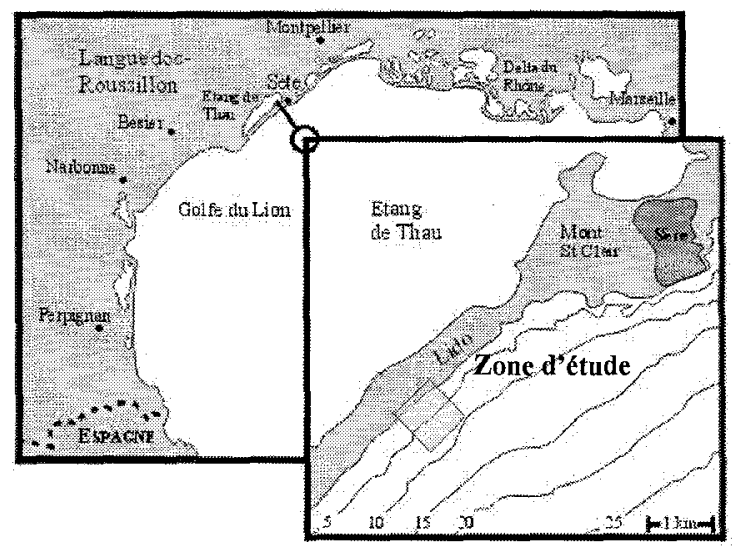

Fig. 1 : localisation de la zone d'étude. Study zone.

Ce site microtidal ( $0,15 \mathrm{~m}$ de marnage) est dominé par les vagues. Les vents dominants proviennent de terre (NW) ou de mer (SE). Ils induisent des variations de niveaux importantes, qui peuvent atteindre $1 \mathrm{~m}$ à la côte. Les vagues créées par les vents de NW sont généralement faibles $(0,3 \mathrm{~m})$ avec des périodes allant de 3 à 4 secondes (mer de vent). Les vagues dominantes induites par les vents de SE. Elles atteignent régulièrement des hauteurs significatives de 2,5 mètres avec des périodes voisines de 8 secondes. La morphologie en pente douce $(1 \%)$ du glacis jusqu'au rivage est entrecoupée par les deux barres. La barre la plus proche du rivage est appelée barre interne, la plus éloignée barre externe. Cette terminologie est reprise pour décrire les deux fosses séparatrices. Le sédiment superficiel est fin à moyen (médiane de 125 à $320 \mu \mathrm{m}$ ), généralement bien trié et sa médiane décroît du bord vers le large.

\subsection{Dispositif expérimental}

L'expérimentation de terrain s'est déroulée du 3 novembre au 3 décembre 2000 (Certain et al., 2001). Le dispositif instrumental se composait de trois courantomètres/houlographes Interocean de type $\mathrm{S} 4$ (DW et $\mathrm{ADW}$ ) fixés sur des structures a- 


\section{VII ${ }^{\text {èmes }}$ Journées Nationales Génie Civil - Génie Côtier, Anglet, France, 15-17 Mai 2002}

magnétiques. Ces instruments mesurent les deux composantes du courant horizontal à une hauteur comprise entre $0,5 \mathrm{~m}$ et $0,9 \mathrm{~m}$ par rapport au fond marin et la pression, à une distance $\mathrm{du}$ fond de $0,4 \mathrm{~m}$ à $0,8 \mathrm{~m}$. Ces données permettent d'évaluer les caractéristiques directionnelles des vagues.

Les données brutes sont acquises à une fréquence de $2 \mathrm{~Hz}$ pendant 18 à 36 minutes toutes les trois heures. Une fois moyennées, elles fournissent les caractéristiques du courant horizontal moyen ainsi que les fluctuations du niveau du plan d'eau statique. Les caractéristiques de l'agitation (hauteur significative et périodes caractéristiques) sont déduites des séries temporelles des fluctuations de pression par analyse spectrale.

La direction de provenance de la houle est déduite de l'analyse des spectres croisés des fluctuations de pression et des deux composantes horizontales du courant. Les S4 étaient disposés sur le glacis et dans les creux inter-barres (respectivement à $-6,0,-4,0$ et $-2,5 \mathrm{~m}$ ) afin d'étudier les conditions de houle à l'entrée du système et sa déformation après passage sur les barres (fig.2).

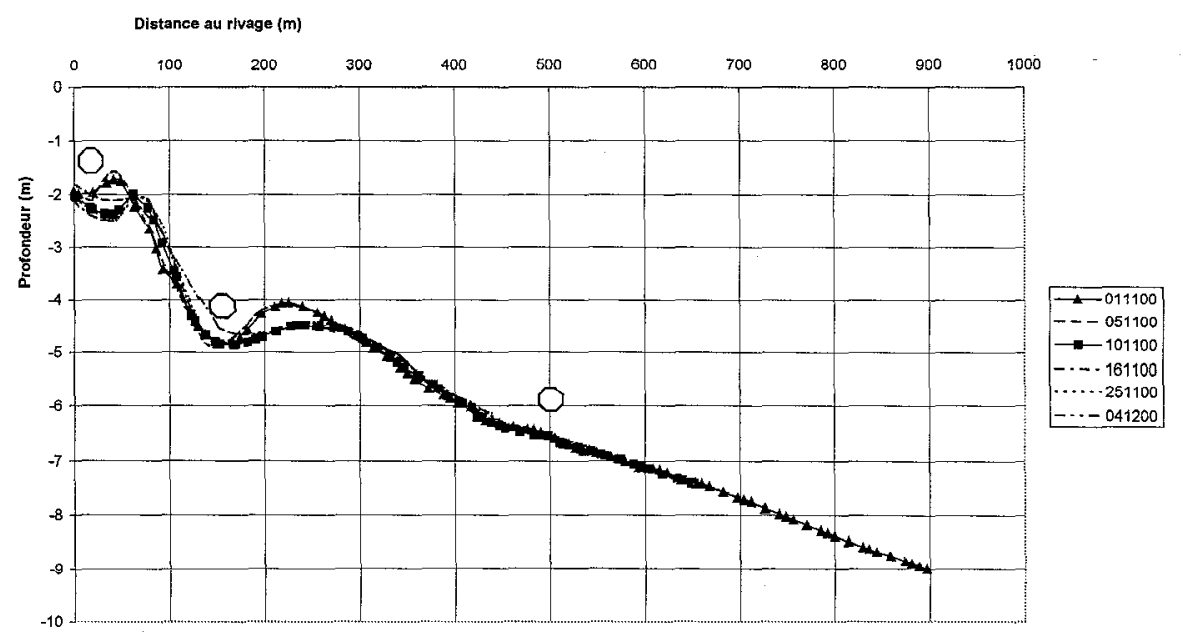

Fig. 2 : le profil transversal instrumenté et sa morphodynamique au cours de la campagne. Morphodynamic of the experimented profile during the study

Lors des tempêtes deux déferlements successifs peuvent se produire au passage des barres. Pour des houles plus petites, les vagues peuvent parvenir à la côte sans déferler ou seulement sur la barre interne. En fonction de ce critère visuel, une typologie des conditions hydrodynamiques présentes sur le site de Sète a été établie. Dix situations sont distinguées (tabl.1).

Seules les situations de maximum de tempêtes et quelques cas mettant en évidence des transferts d'énergies seront présentés dans cet article. L'analyse et la comparaison de ces situations sont effectuées à l'aide de FFT réalisées sur 512 valeurs avec des fréquences de coupure entre $0,003 \mathrm{~Hz}$ et $0,40 \mathrm{~Hz}(300 \mathrm{~s}-2,5 \mathrm{~s})$. Selon Munk (1951, in O.M.M., 1989), la limite entre vagues gravitaires et infragravitaires se situe autour de $30 \mathrm{~s}$. Les vagues de gravité ont une période comprise entre $1 \mathrm{~s}$ et $30 \mathrm{~s}$, tandis que la période des houles infragravitaires se situe entre $30 \mathrm{~s}$ et $300 \mathrm{~s}$. Les fréquences de coupure adoptées sont proches de ces limites tout en choisissant une coupure haute inférieure à celle proposée par Munk afin de limiter le bruitage.

Le suivi bathymétrique, qui permet de contrôler la morphodynamique associée aux coups de mer observés (fig.2), a été réalisé à l'aide d'un sondeur Tritech ST 500 relié à un PC portable embarqué sur un zodiac. Ce modèle de sondeur donne une précision centimétrique de 
la mesure verticale. L'incertitude de la mesure est de $+/-5$ centimètres. Le PC est aussi relié à un GPS différentiel (Sercel), placé au sommet de la tige du sondeur. Un positionnement au mètre près est obtenu dans le plan horizontal. Le pas d'acquisition est métrique.

\begin{tabular}{|c|c|}
\hline $\begin{array}{l}\text { Situations rencontrées } \\
\text { classées suivant l'agitation } \\
\text { croissante du milieu }\end{array}$ & Type de conditions hydrodynamiques \\
\hline Situation 1 & Mer de vent sans houle associée \\
\hline Situation 2 & $\begin{array}{l}\text { Oscillations de grande longueur d'onde et de grande période } \\
(10 \mathrm{~s}) \text { mais de très faible amplitude }(0,05 \mathrm{~m})\end{array}$ \\
\hline Situation 3 & Petite houle $(0,2 \mathrm{~m})$ non déferlante \\
\hline Situation 4 & Petite houle $(0,2 \mathrm{~m})$ non déferlante et mer de vent \\
\hline Situation 5 & Petite houle à déferlement occasionnel \\
\hline Situation 6 & Petite houle déferlante sur la barre interne \\
\hline Situation 7 & Amortissement de la houle de tempête avec déferlement \\
\hline Situation 8 & Grosse houle plongeante avec vent de terre \\
\hline Situation 9 & $\begin{array}{l}\text { Mer de vent NE et clapot haché pré-tempête ; Déferlement } \\
\text { généralisé d'une houle courte avec mer de vent }\end{array}$ \\
\hline Situation 10 & Tempête \\
\hline
\end{tabular}

Tableau 1 : Types de conditions hydrodynamiques rencontrées sur le site de Sète

\section{3-Résultats}

La figure 3 représente les hauteurs significatives de la houle en chacun des trois points de mesure : le glacis, la fosse externe et la fosse interne. Les trois premières tempêtes ont des hauteurs significatives très proches $(2,5 \mathrm{~m})$, la quatrième est de plus faible intensité $(2 \mathrm{~m})$. Dans la fosse externe, après un premier déferlement sur la barre externe, les hauteurs significatives sont semblables pour tous les coups de mer $(2 \mathrm{~m})$. Dans la fosse interne, après un second déferlement sur la barre interne, les hauteurs sont très proches pour l'ensemble des coups de mer, entre 0,7 et $1 \mathrm{~m}$.

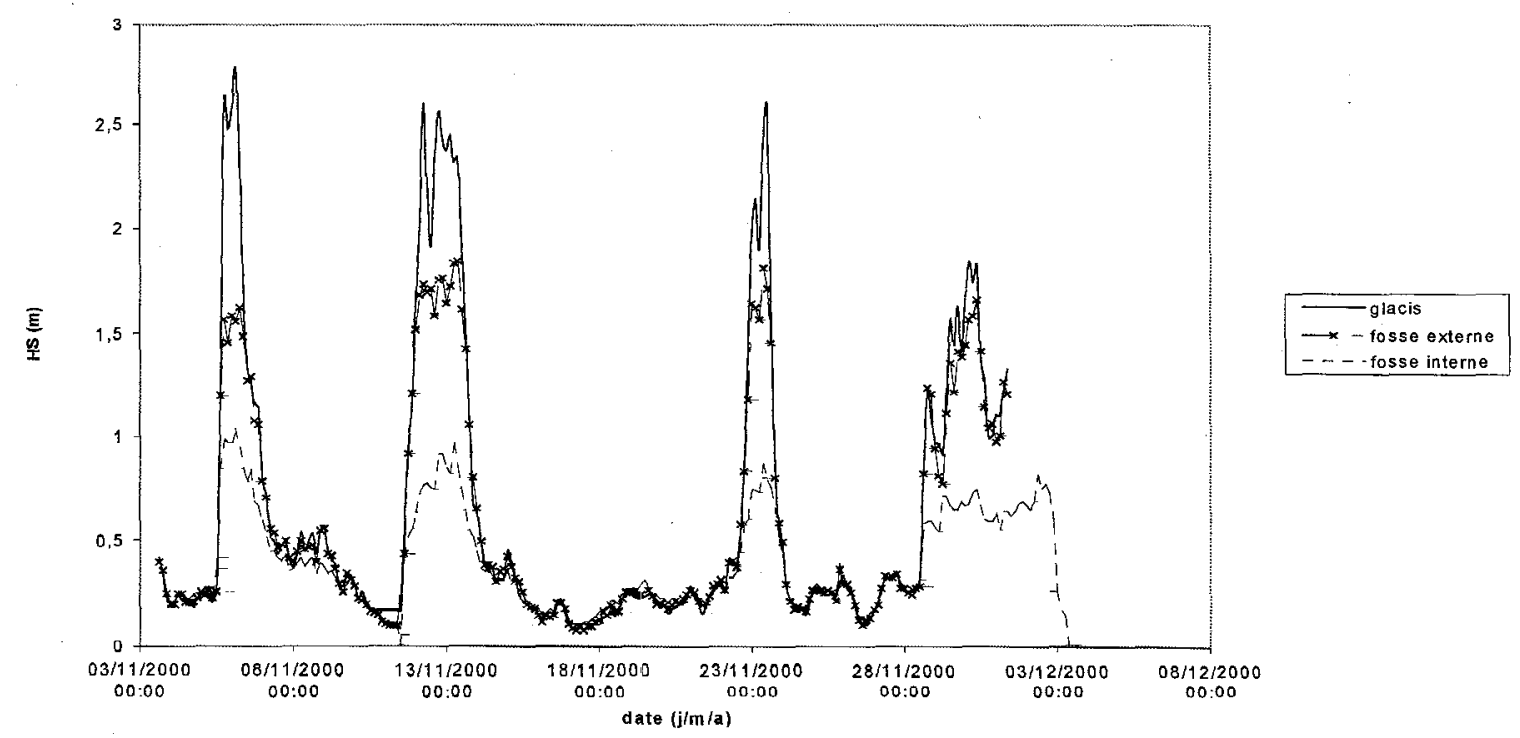

Fig. 3 : Hauteurs significatives de la houle au cours de la campagne de mesure, du 3 novembre au 3 décembre 2000. Significant swell height during study. 
L'observation visuelle des séries temporelles de niveau est toutefois insuffisante pour mettre en évidence la spécificité des spectres des vagues pour chacune de ces tempêtes. La figure 4 représente les résultats de FFT pour chacun des maxima des quatre tempêtes (fig.3). Les différentes fréquences du spectre de houle présentes au moment de l'analyse dans un intervalle de 0,003 à $0,4 \mathrm{~Hz}$ sont exprimées en fonction de la densité spectrale d'énergie en $\mathrm{m}^{2} / \mathrm{Hz}$. Ce cas correspond à la situation 10 de la classification élaborée dans le tableau 1 .

La première tempête est la tempête qui est la plus énergétique avec un pic principal à $20 \mathrm{~m}^{2} / \mathrm{Hz}$, par rapport à la deuxième et troisième tempêtes qui ont un pic à $11 \mathrm{~m}^{2} / \mathrm{Hz}$ et la tempête 4 à $6 \mathrm{~m}^{2} / \mathrm{Hz}$. C'est aussi vrai si on considère l'intégrale des pics au lieu de leurs maximums. La densité spectrale dans la fosse externe est par contre sensiblement égale pour chacune des tempêtes si on se place après les points de déferlement dans la fosse externe (autour de $5 \mathrm{~m}^{2} / \mathrm{Hz}$ ) et dans la fosse interne (autour de $1 \mathrm{~m}^{2} / \mathrm{Hz}$ ) (Guza et Thornton, 1982). Dans le domaine infragravitaire, la description se complique. La première tempête produit làencore le maximum d'énergie dans ce domaine avec $0,8 \mathrm{~m}^{2} / \mathrm{Hz}$, contre 0,2 à $0,4 \mathrm{~m}^{2} / \mathrm{Hz}$ pour les tempêtes suivantes.

Pour la tempête 1 , l'énergie est constante dans chacun des compartiments alors que le pic principal diminue, transférant certainement de l'énergie vers ses sous-harmoniques. Pour les autres tempêtes il est difficile d'interpréter l'évolution du domaine infragravitaire tant les résultats sont complexes et divers. Pour mieux observer ces transferts, il convient donc de se placer dans des situations moins bruitées, où le spectre est moins large et présente plus d'uniformité de valeurs de densité spectrale dans les différents domaines.

La figure 5 suit cette approche et reprend la même méthode analytique que celle exposée dans le paragraphe précédent. Cette fois, quatre situations de la classification, moins agitées que celle étudiée précédemment, sont analysées, les situations $2,4,6$ et 8 . Cette sélection est guidée par l'intérêt de ces cas en terme de transfert d'énergie du pic principal vers les harmoniques. Pour une meilleure lisibilité, tous les graphiques ne sont pas à la même échelle.

La situation 2 est celle d'une petite houle non déferlante. Elle met en évidence un élargissement du pic principal $(0,084 \mathrm{~Hz})$ au passage de la barre externe (sans déferlement donc). La largeur du pic principal qui regroupe deux pics $(0,075$ et $0,1 \mathrm{~Hz})$, présents dans la fosse externe, est en relation avec la valeur du pic de l'infragravité à $0,04 \mathrm{~Hz}$ dans la fosse interne. On suppose donc qu'il y a eu transfert d'énergie du domaine gravitaire vers les basses fréquences. L'infragravité est maximum au bord et minimum au niveau du glacis.

La situation 4 est sensiblement identique à la précédente bien que la densité spectrale dans les domaines infragravitaires soit plus faible. Par contre on observe une forte énergie concentrée au niveau de la mer de vent entre 0,25 et $0,4 \mathrm{~s}$.

La situation 6 est nettement plus énergétique, elle se situe à la fin de la première tempête. Sur le glacis, on a deux pics principaux à 0,13 et $0,10 \mathrm{~Hz}$, que l'on retrouve dans la fosse externe. La densité spectrale des pics principaux est inchangée, aucun déferlement ne s'est produit sur la barre externe. Mais, commence à apparaître de l'énergie infragravitaire à une fréquence correspondant à l'interaction différence des deux pics libres principaux $(0,03$ $\mathrm{Hz}$ ) dans la fosse externe. Lors du passage de la barre interne, la houle déferle faisant chuter la densité spectrale d'énergie (dsp) des pics principaux. L'énergie infragravitaire, elle, augmente. On remarquera aussi qu'entre le cas où le déferlement commence à se produire et un maximum de tempête, il y a un facteur 10 en terme de dsp.

La figure 2 illustre les changements morphologiques des barres associés à ces coups de mer. Les levés ont été effectués avant et après chacun des épisodes de tempêtes. On constate que la première tempête, qui se produit le 6 novembre 2000, induit un recul net des deux barres (comparaison des levés du 5 novembre et du 10 novembre 2000). Ce recul est 

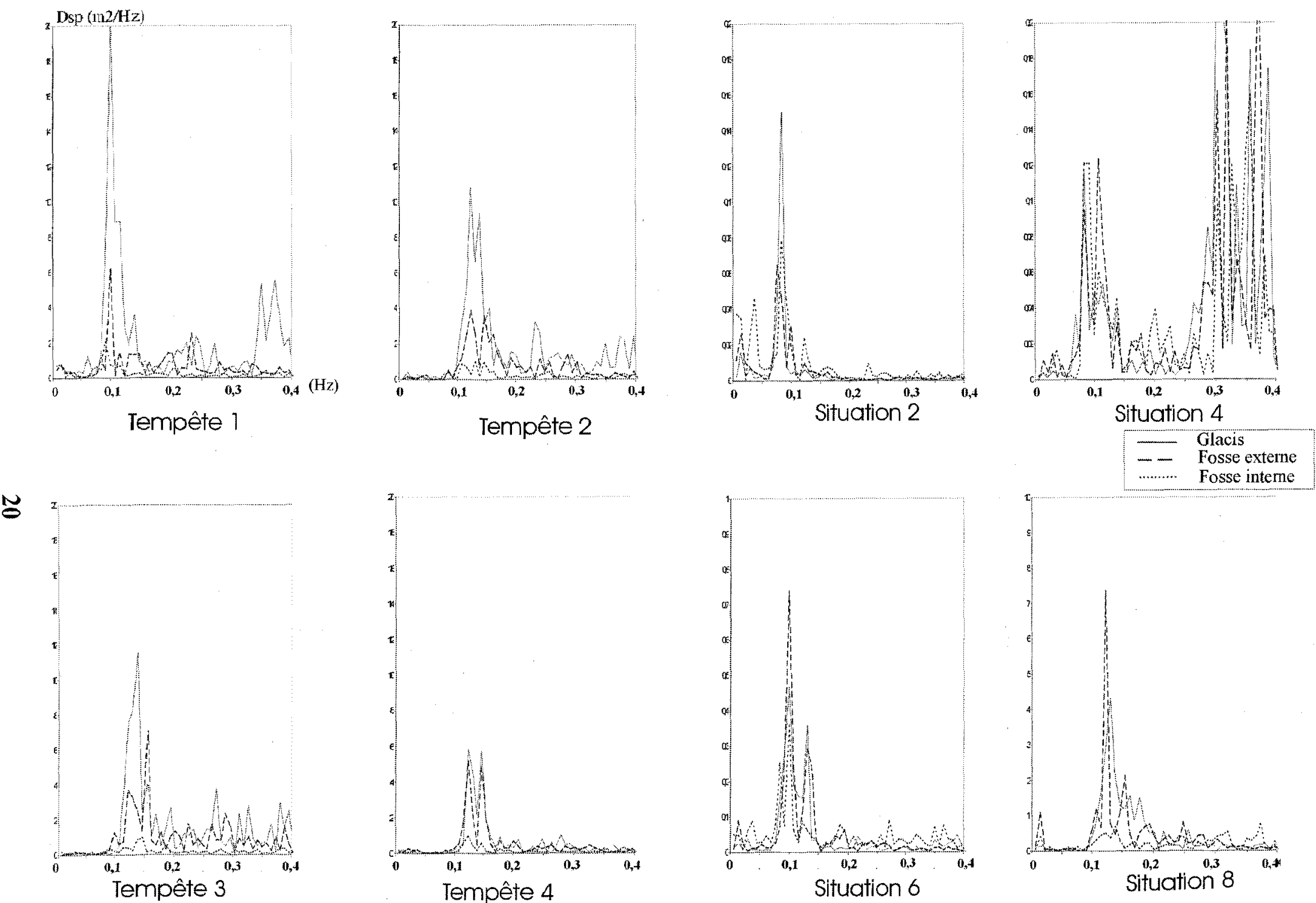

|

Fig.4: maximum des 4 tempêtes observées (storms maxima)

Fig. 5: analyse de situations types [different cases) 


\section{VII ${ }^{\grave{m} e s}$ Journées Nationales Génie Civil-Génie Côtier, Anglet, France, 15-17 Mai 2002}

particulièrement marqué au niveau de la barre externe décrite habituellement comme très stable. Une fois cette mise à l'équilibre hydrodynamique effectuée, les tempêtes suivantes, de hauteurs significatives pourtant comparables, $n$ 'induisent aucun changement morphologique significatif.

\section{4-Discussion/conclusion}

Ces résultats mettent en avant le rôle primordial des barres sédimentaires dans la dissipation et la redistribution spectrale de l'énergie de la houle à l'approche de la côte.

Dans le cas sans déferlement (situation 2 et 4 ) on observe que le passage du train d'ondes d'un milieu de grande profondeur vers un milieu de faible profondeur entraîne des modifications au niveau du spectre d'énergie. On assiste, en particulier, à des transferts d'énergie vers les sous-harmoniques par des interactions différences des harmoniques libres. La présence d'infragravité induite est maximum à la côte, conformément à la théorie (Aagard and Greenwood, 1995).

Dans le cas avec déferlement (situations 6,8 et 10), on constate que le déferlement accentue ces modifications. Pour les déferlements faiblement énergétiques ( 6 et 8 ), on retrouve distinctement les transferts vers les sous-harmoniques à la différence que les vagues gravitaires subissant le déferlement transfèrent beaucoup plus d'énergie au domaine infragravitaire. Pour les cas de tempête (situation 10), on peut mesurer une forte atténuation entre l'entrée du système (glacis) et le bord (fosse interne), sauf dans le domaine des ondes infragravitaires qui présentent un comportement différent de celui des deux autres domaines au cours de la transformation de la vague.

Ce comportement peut aussi être très variable suivant les tempêtes observées. On voit cependant que les barres agissent alors comme des régulateurs ou transformateurs d'énergie. $\mathrm{Si}$ on considère les hauteurs significatives ou les densités spectrales d'énergie, on remarque qu'elles sont du même ordre de grandeur dans les différents compartiments. Quelles que soient les hauteurs ou l'énergie des vagues sur le glacis, on aura toujours la même hauteur ou dsp après déferlement sur la barre externe. Si l'énergie est très forte sur le glacis, comme pour la tempête 1 , alors qu'elle est trois fois inférieure pour la tempête 4 , les valeurs de ces deux cas pour la fosse externe sont identiques. Dans un cas il y a eu déferlement pour dissiper de l'énergie et déplacement de sable. Dans l'autre cas il n'y a pas eu de déferlement et la dsp est la même après le passage de la barre. Ce schéma est reproductible sur la barre interne.

La méthodologie utilisée montre toute son efficacité pour expliquer des changements morphologiques et analyser les processus hydrodynamiques à l'origine de ces changements. Cela a été déjà illustré avec l'analyse de la première tempête dont on a pu voir sa particularité. Le travail devra être poursuivi avec le couplage des observations morphologiques effectuées en même temps que les mesures.

\section{Références}

Aagard and Grenwood, 1995. Suspended sediment transport and morphological response on a dissipative beach. Continental Shelf Research, 15, pp 1061-1086.

Abadie, S., 1998. Modélisation numérique du déferlement plongeant par méthode VOF, Thèse de $3^{\text {ème }}$ cycle, Université de Bordeaux I.

Cadène, A-L., 2000. Dynamique des ondes infragravitaires générées par la houle en zone littorale. Thèse de $3^{\text {ème }}$ cycle, Institut national polytechnique de Toulouse, $239 \mathrm{p}$. 
Certain, R., Barusseau, J. P., Capobianco, R., Meuret, A., Rey, V., Dulou, C., Stepanian, A., Levoy, F. and Howa, H.Bottom and shoreline evolutions under wave actions at a french Mediterranean site : The beach of Sète. MEDCOASTO1, The fifth International Conference on the Mediterraneen Coastal Environment, 23-27 October 2001, Hamammet, Tunisia

Dulou, C., 2000a. Interactions houle-sédiments: application à la formation des barres littorales. Thèse de $3^{\text {ème }}$ cycle, Univ. de Provence, $188 \mathrm{p}$.

Dulou, C., Rey, V. et Belzons, M., 2000b. Etude expérimentale de l'évolution spatiotemporelle du couplage onde-fond sédimentaire en zone de déferlement. Comptes rendus des $6^{\text {èmes }}$ Journées Génie Côtier-Génie Civil, Caen, Mai 2000, I, pp 137-146.

Elgar, S., Guza, R.T., Raubeiheimer, B., Herbers, T.H.C and Gallagher, E.L., 1997. Spectral evolution of shoaling and breaking waves on a barred beach. J. Geophys. Res., 102, pp. 15797-15805.

Guza, R.T., and Thorton, E.B.,1982. Swash oscillations on a natural beach. J. of Geophys. Research, 87, 483-491.

Holman, R.A. and Sallenger, A.H., 1993. Sand bar generation: a discussion of the Duck experiment series. Journal of Coastal research, vol. 87, $\mathrm{N}^{\circ} . \mathrm{C} 1$.

Madsen P.A., Sørensen O.R. and Schäffer H.A, 1997. Surf zone dynamics simulated by a Boussinesq type model. Part I. Model description and cross-shore motion of regular waves, Coastal Engineering, Vol 32, pp 255-287.

O'Hare, T.J. and Davis, A.G., 1993. Sand bar formation beneath partially standing waves: laboratory experiment and model simulation. Cont. Shelf Res., 13, pp 1149-1181.

Organisation Météorologique Mondiale, 1989. Guide de l'analyse et de la prévision des vagues. Rapport OMM No 702,222 p. 